\title{
Correlation of Fundus Findings in Retinal Vein Occlusion (RVO) with Serum Lipid Profile.
}

\author{
Dr.Himangshu Das, Dr. J.J.Kuli \\ (Department of Ophthalmology, Assam Medical College \& Hospital, Dibrugarh, India)
}

\begin{abstract}
Purpose:To determine the serum lipid levels in RVO patients \&correlate it with the individual fundoscopic findings.

Setting: Assam Medical College \& Hospital,Dibrugarh.

Study design:1-year hospital based observational study

Patients and Methods:We determined the serum lipid levels of 47 cases of RVO (15 CRVO, 32 BRVO) in Autoanalyzer in advanced clinical biochemistry laboratory. The fundoscopic findings were documented by fundus photography and FFA. There were no controls in our study.

Results:On correlating the various fundoscopic findings with serum lipid profile, the correlation of VLDL with disc edema and macular edema was found to be statistically highly significant. Correlation of decreased HDL with retinal and disc neovascularization was found to be statistically significant.

Conclusion:Increased VLDL may play some role in the development of macular and disc edema, while decreased HDL in the development of neovascularization in RVO patients.
\end{abstract}

\section{Introduction}

Venous occlusive disease of the retina is the second most common retinal vascular disorder, after diabetic retinopathy. RVO is an important cause of visual loss, particularly in middle age.

RVO have a characteristic, although somewhat variable, appearance with intraretinalhaemorrhage, tortuous and dilated retinal veins, exudates, cotton wool spots, retinal edema and occasionally optic disc swelling. Possible explanation for the association of retinal vein occlusion and hyperlipidemia may be through effects on plasma viscosity or alteration in platelet function with release of platelet specific protein (beta thromboglobulin and platelet factor 4 thereby predisposing to platelet aggregation and thrombosis. Also, younger women are at a higher risk of developing RVO. This may be due to the effect of oral contraceptive drugs on serum lipids which increase plasma viscosity. ${ }^{1}$

Studies have suggested high density lipoproteins (HDL) cholesterol may protect against microvascular disease, whereas low density lipoprotein (LDL) cholesterol may predispose to it. ${ }^{2}$ Macular edema in ischemic CRVO is often severe and massive lipid exudation can occur in the macular region, especially in patients with elevated triglyceride levels. ${ }^{3}$ Lipemiaretinalis is an unusual retinal manifestation of hyperlipidemia and is thought to be directly correlated with the serum triglyceride level. ${ }^{4}$

\section{Aims And Objectives:}

\section{Materials And Methods}

The aims of the study were:

(1) Determination of serum lipid levels in known cases of RVO.

(2) Correlation of fundoscopic findings with serum lipid levels.

\section{Source Of Data:}

Clinically diagnosed cases of retinal venous occlusion (RVO) attending the outpatient department, Department of Ophthalmology, Assam Medical College and Hospital, Dibrugarh.

\section{Method Of Collection Of Data:}

47 cases of RVO (15 CRVO, 32 BRVO) were included in the study. The cases were diagnosed clinically by direct and indirect ophthalmoscopy. FFA was done for documentation and treatment purpose. Fundus findings were recorded.Then the following tests were done:

1. Routine blood investigations

2. Blood pressure

3. Blood sugar

4. Serum lipid profile.

Inclusion Criteria:

> Patients with CRVO, HRVO \& BRVO

$>$ Gender-both

$>$ Age 20 years and above 


\section{Exclusion Criteria:}

$>$ Cataract \& other media opacities.

$>$ Other associated ocular morbidity like uveitis.

$>$ Patients not willing to undergo any investigations or follow up.

$>$ Old cases of retinal vein occlusion

$>$ History of smoking or alcohol intake

$>$ Patients with drug history of thiazide diuretics, beta-blockers, anti-retroviral drugs, oral contraceptives, glucocorticoids which are known to cause dyslipidemia.

\section{Statistical Analysis:}

The statistical significance was evaluated using chi square test. $\mathrm{p}$ value $<0.05$ and $<0.01$ were considered as statistically significant and highly significant respectively whereas $p$ value $>0.05$ was considered not significant.

\section{Results And Observations}

Table-1:Correlation of Lipid Profile with Diffuse Haemorrhage

\begin{tabular}{|c|c|c|c|c|c|c|}
\hline \multicolumn{2}{|l|}{ LIPID PROFILE } & \multicolumn{2}{|c|}{$\begin{array}{l}\begin{array}{l}\text { WITH } \\
(\mathrm{n}=15)\end{array}\end{array}$} & \multicolumn{2}{|c|}{$\begin{array}{l}\text { WITHOUT } \\
(\mathrm{n}=32)\end{array}$} & $\mathrm{p}$ value \\
\hline \multirow{3}{*}{ Triglycerides } & & & & & & \multirow{3}{*}{$>0.05$} \\
\hline & Normal & 6 & 40.00 & 17 & 53.13 & \\
\hline & Elevated & 9 & 60.00 & 15 & 46.88 & \\
\hline \multirow[t]{2}{*}{ Total Cholesterol } & Normal & 8 & 53.33 & 16 & 50.00 & \multirow[t]{2}{*}{$>0.05$} \\
\hline & Elevated & 7 & 46.67 & 16 & 50.00 & \\
\hline \multirow[t]{2}{*}{ LDL } & Normal & 9 & 60.00 & 15 & 46.88 & \multirow[t]{2}{*}{$>0.05$} \\
\hline & Elevated & 6 & 40.00 & 17 & 53.13 & \\
\hline \multirow[t]{2}{*}{ HDL } & Normal & 10 & 66.67 & 26 & 81.25 & \multirow[t]{2}{*}{$>0.05$} \\
\hline & Decreased & 5 & 33.33 & 6 & 18.75 & \\
\hline \multirow[t]{2}{*}{ VLDL } & Normal & 12 & 80.00 & 28 & 87.50 & \multirow[t]{2}{*}{$>0.05$} \\
\hline & Elevated & 3 & 20.00 & 4 & 12.50 & \\
\hline
\end{tabular}

Table-2:Correlation of Lipid Profile with SectoralHaemorrhage

\begin{tabular}{|c|c|c|c|c|c|c|}
\hline \multicolumn{2}{|l|}{ LIPID PROFILE } & \multicolumn{2}{|c|}{$\begin{array}{l}\text { WITH } \\
(\mathrm{n}=32)\end{array}$} & \multicolumn{2}{|c|}{$\begin{array}{l}\text { WITHOUT } \\
(\mathrm{n}=15)\end{array}$} & \multirow[t]{2}{*}{$\mathrm{p}$ value } \\
\hline & & $\mathrm{N}$ & $\%$ & $\mathrm{n}$ & $\%$ & \\
\hline \multirow[t]{2}{*}{ Triglycerides } & Normal & 17 & 53.13 & 6 & 40.00 & \multirow[t]{2}{*}{$>0.05$} \\
\hline & Elevated & 15 & 46.88 & 9 & 60.00 & \\
\hline \multirow[t]{2}{*}{ Total Cholesterol } & Normal & 16 & 50.00 & 8 & 53.33 & \multirow[t]{2}{*}{$>0.05$} \\
\hline & Elevated & 16 & 50.00 & 7 & 46.67 & \\
\hline \multirow[t]{2}{*}{ LDL } & Normal & 15 & 46.88 & 9 & 60.00 & \multirow[t]{2}{*}{$>0.05$} \\
\hline & Elevated & 17 & 53.13 & 6 & 40.00 & \\
\hline \multirow[t]{2}{*}{ HDL } & Normal & 26 & 81.25 & 10 & 66.67 & \multirow[t]{2}{*}{$>0.05$} \\
\hline & Decreased & 6 & 18.75 & 5 & 33.33 & \\
\hline \multirow[t]{2}{*}{ VLDL } & Normal & 28 & 87.50 & 12 & 80.00 & \multirow[t]{2}{*}{$>0.05$} \\
\hline & Elevated & 4 & 12.50 & 3 & 20.00 & \\
\hline
\end{tabular}

Table-3:Correlation Of Lipid Profile with Cotton Wool Spot

\begin{tabular}{|c|c|c|c|c|c|c|}
\hline \multicolumn{2}{|l|}{ LIPID PROFILE } & \multicolumn{2}{|c|}{$\begin{array}{l}\text { WITH } \\
(\mathrm{n}=29)\end{array}$} & \multicolumn{2}{|c|}{$\begin{array}{l}\text { WITHOUT } \\
(\mathrm{n}=18)\end{array}$} & $\mathrm{p}$ value \\
\hline \multirow{3}{*}{ Triglycerides } & & & & & & \multirow{3}{*}{$>0.05$} \\
\hline & Normal & 14 & 48.28 & 9 & 50.00 & \\
\hline & Elevated & 15 & 51.72 & 9 & 50.00 & \\
\hline \multirow[t]{2}{*}{ Total Cholesterol } & Normal & 16 & 55.17 & 8 & 44.44 & \multirow[t]{2}{*}{$>0.05$} \\
\hline & Elevated & 13 & 44.83 & 10 & 55.56 & \\
\hline \multirow[t]{2}{*}{ LDL } & Normal & 14 & 48.28 & 10 & 55.56 & \multirow[t]{2}{*}{$>0.05$} \\
\hline & Elevated & 15 & 51.72 & 8 & 44.44 & \\
\hline \multirow[t]{2}{*}{ HDL } & Normal & 20 & 68.97 & 16 & 88.89 & \multirow[t]{2}{*}{$>0.05$} \\
\hline & Decreased & 9 & 31.03 & 2 & 11.11 & \\
\hline \multirow[t]{2}{*}{ VLDL } & Normal & 24 & 82.76 & 16 & 88.89 & \multirow[t]{2}{*}{$>0.05$} \\
\hline & Elevated & 5 & 17.24 & 2 & 11.11 & \\
\hline
\end{tabular}


Correlation of Funfus Findings in Retinal Vein Occlusion (Rvo) with Serun Lipid Profile.

Table-4:Correlation Of Lipid Profile with Exudates

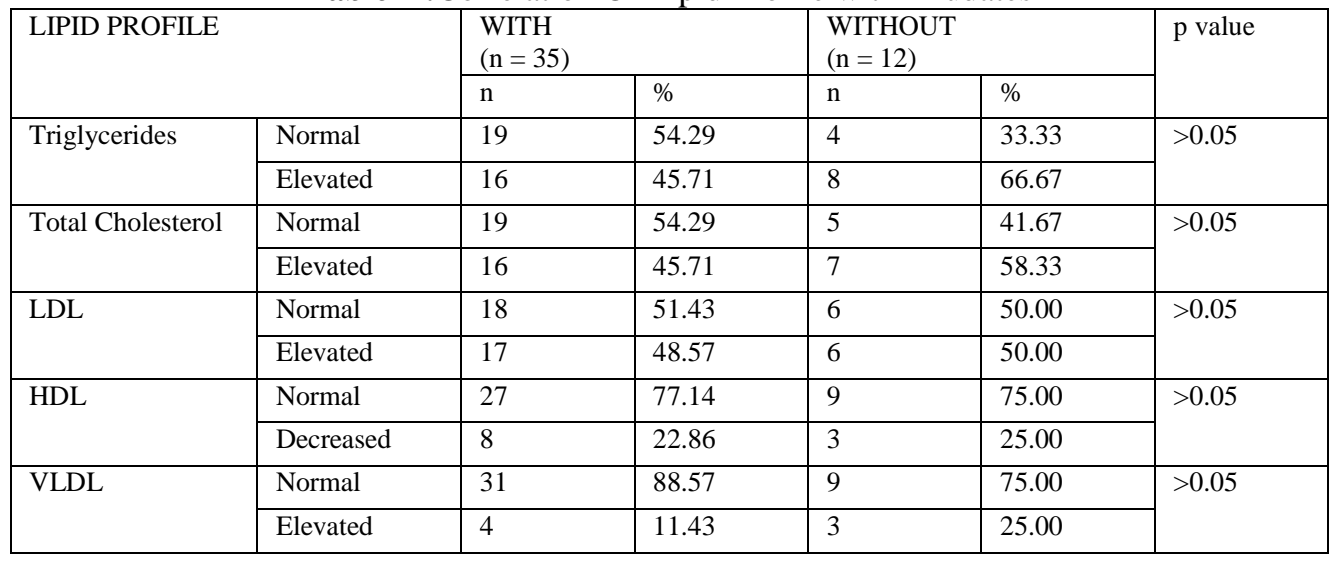

Table-5:Correlation Of Lipid Profile with Disc Edema

\begin{tabular}{|c|c|c|c|c|c|c|}
\hline \multirow[t]{2}{*}{ LIPID PROFILE } & & \multicolumn{2}{|c|}{$\begin{array}{l}\text { WITH } \\
(\mathrm{n}=18)\end{array}$} & \multicolumn{2}{|c|}{$\begin{array}{l}\text { WITHOUT } \\
(\mathrm{n}=29)\end{array}$} & \multirow[t]{2}{*}{$\mathrm{p}$ value } \\
\hline & & $\mathrm{n}$ & $\%$ & $\mathrm{n}$ & $\%$ & \\
\hline \multirow[t]{2}{*}{ Triglycerides } & Normal & 8 & 44.44 & 15 & 51.72 & \multirow[t]{2}{*}{$>0.05$} \\
\hline & Elevated & 10 & 55.56 & 14 & 48.28 & \\
\hline \multirow[t]{2}{*}{ Total Cholesterol } & Normal & 11 & 61.11 & 13 & 44.83 & \multirow[t]{2}{*}{$>0.05$} \\
\hline & Elevated & 7 & 38.89 & 16 & 55.17 & \\
\hline \multirow[t]{2}{*}{ LDL } & Normal & 8 & 44.44 & 16 & 55.17 & \multirow[t]{2}{*}{$>0.05$} \\
\hline & Elevated & 10 & 55.56 & 13 & 44.83 & \\
\hline \multirow[t]{2}{*}{ HDL } & Normal & 12 & 66.67 & 24 & 82.76 & \multirow[t]{2}{*}{$>0.05$} \\
\hline & Decreased & 6 & 33.33 & 5 & 17.24 & \\
\hline \multirow[t]{2}{*}{ VLDL } & Normal & 12 & 66.67 & 28 & 96.55 & \multirow[t]{2}{*}{$<0.01$} \\
\hline & Elevated & 6 & 33.33 & 1 & 3.45 & \\
\hline
\end{tabular}

Table-6:Correlation Of Lipid Profile with Retinal Neovascularization

\begin{tabular}{|c|c|c|c|c|c|c|}
\hline \multirow{2}{*}{\multicolumn{2}{|c|}{ LIPID PROFILE }} & \multicolumn{2}{|c|}{$\begin{array}{l}\text { WITH } \\
(\mathrm{n}=9)\end{array}$} & \multicolumn{2}{|c|}{$\begin{array}{l}\text { WITHOUT } \\
(\mathrm{n}=38)\end{array}$} & \multirow[t]{2}{*}{$\mathrm{p}$ value } \\
\hline & & $\mathrm{n}$ & $\%$ & $\mathrm{n}$ & $\%$ & \\
\hline \multirow[t]{2}{*}{ Triglycerides } & Normal & 6 & 66.67 & 17 & 44.74 & \multirow[t]{2}{*}{$>0.05$} \\
\hline & Elevated & 3 & 33.33 & 21 & 55.26 & \\
\hline \multirow[t]{2}{*}{ Total Cholesterol } & Normal & 7 & 77.78 & 17 & 44.74 & \multirow[t]{2}{*}{$>0.05$} \\
\hline & Elevated & 2 & 22.22 & 21 & 55.26 & \\
\hline \multirow[t]{2}{*}{ LDL } & Normal & 4 & 44.44 & 20 & 52.63 & \multirow[t]{2}{*}{$>0.05$} \\
\hline & Elevated & 5 & 55.56 & 18 & 47.37 & \\
\hline \multirow[t]{2}{*}{ HDL } & Normal & 5 & 55.56 & 33 & 86.84 & \multirow[t]{2}{*}{$<0.05$} \\
\hline & Decreased & 4 & 44.44 & 5 & 13.16 & \\
\hline \multirow[t]{2}{*}{ VLDL } & Normal & 7 & 77.78 & 33 & 86.84 & \multirow[t]{2}{*}{$>0.05$} \\
\hline & Elevated & 2 & 22.22 & 5 & 13.16 & \\
\hline
\end{tabular}

Table-7:Correlation Of Lipid Profile with Disc Neovascularization

\begin{tabular}{|c|c|c|c|c|c|c|}
\hline \multicolumn{2}{|l|}{ LIPID PROFILE } & \multicolumn{2}{|c|}{$\begin{array}{l}\text { WITH } \\
(n=2)\end{array}$} & \multicolumn{2}{|c|}{$\begin{array}{l}\text { WITHOUT } \\
(\mathrm{n}=45)\end{array}$} & \multirow[t]{2}{*}{$\mathrm{p}$ value } \\
\hline & & $\mathrm{n}$ & $\%$ & $\mathrm{n}$ & $\%$ & \\
\hline \multirow[t]{2}{*}{ Triglycerides } & Normal & 1 & 50.00 & 22 & 48.89 & \multirow[t]{2}{*}{$>0.05$} \\
\hline & Elevated & 1 & 50.00 & 23 & 51.11 & \\
\hline \multirow[t]{2}{*}{ Total Cholesterol } & Normal & 1 & 50.00 & 23 & 51.11 & \multirow[t]{2}{*}{$>0.05$} \\
\hline & Elevated & 1 & 50.00 & 22 & 48.89 & \\
\hline \multirow[t]{2}{*}{ LDL } & Normal & 0 & 0.00 & 24 & 53.33 & \multirow[t]{2}{*}{$>0.05$} \\
\hline & Elevated & 2 & 100.00 & 21 & 46.67 & \\
\hline \multirow[t]{2}{*}{ HDL } & Normal & 0 & 0.00 & 36 & 80.00 & \multirow[t]{2}{*}{$<0.01$} \\
\hline & Decreased & 2 & 100.00 & 9 & 20.00 & \\
\hline
\end{tabular}


Correlation of Funfus Findings in Retinal Vein Occlusion (Rvo) with Serun Lipid Profile.

\begin{tabular}{|l|l|l|l|l|l|l|}
\hline VLDL & Normal & 1 & 50.00 & 39 & 86.67 & \multirow{2}{*}{$>0.05$} \\
\cline { 2 - 6 } & Elevated & 1 & 50.00 & 6 & 13.33 & \\
\hline
\end{tabular}

Table-8:Correlation Of Lipid Profile with Macular Edema

\begin{tabular}{|c|c|c|c|c|c|c|}
\hline \multicolumn{2}{|l|}{ LIPID PROFILE } & \multicolumn{2}{|c|}{$\begin{array}{l}\text { WITH } \\
(\mathrm{n}=42)\end{array}$} & \multicolumn{2}{|c|}{$\begin{array}{l}\text { WITHOUT } \\
(\mathrm{n}=45)\end{array}$} & \multirow[t]{2}{*}{$\mathrm{p}$ value } \\
\hline & & $\mathrm{n}$ & $\%$ & $\mathrm{n}$ & $\%$ & \\
\hline \multirow[t]{2}{*}{ Triglycerides } & Normal & 21 & 50.00 & 3 & 60.00 & \multirow[t]{2}{*}{$>0.05$} \\
\hline & Elevated & 21 & 50.00 & 2 & 40.00 & \\
\hline \multirow[t]{2}{*}{ Total Cholesterol } & Normal & 21 & 50.00 & 3 & 60.00 & \multirow[t]{2}{*}{$>0.05$} \\
\hline & Elevated & 21 & 50.00 & 2 & 40.00 & \\
\hline \multirow[t]{2}{*}{ LDL } & Normal & 21 & 50.00 & 3 & 60.00 & \multirow[t]{2}{*}{$>0.05$} \\
\hline & Elevated & 21 & 50.00 & 2 & 40.00 & \\
\hline \multirow[t]{2}{*}{ HDL } & Normal & 32 & 76.19 & 4 & 80.00 & \multirow[t]{2}{*}{$>0.05$} \\
\hline & Decreased & 10 & 23.81 & 1 & 20.00 & \\
\hline \multirow[t]{2}{*}{ VLDL } & Normal & 37 & 88.10 & 1 & 20.00 & \multirow[t]{2}{*}{$<0.001$} \\
\hline & Elevated & 5 & 11.90 & 4 & 80.00 & \\
\hline
\end{tabular}

\section{Discussion}

In our study a significantly high proportion of patients had abnormal lipid values. Out of the 47 cases, more than $65 \%$ cases occurred in the age group 50-69 years, and maximum number of patients with abnormal lipid profile was found in the same age group. This gives a positive correlation between abnormal lipid profile and occurrence of retinal vein occlusion.

- Diffuse hemorrhage was found in 15 cases of RVO. Triglyceride was found to be elevated in $60 \%$ of these cases. Sectoral hemorrhage was found in 32 cases of RVO. Total cholesterol is elevated in 50\%, while LDL is elevated in $53.13 \%$ of these cases. Correlation of lipid profile with hemorrhage was found to be statistically not significant.

- Cotton wool spots were found in 29 cases of RVO. Triglycerides and LDL were found to be elevated in more than $50 \%$ of these cases. Exudates were found in 35 cases. The correlation of lipid profile with cotton wool spots and exudates in RVO patients was found to be statistically not significant.

- Disc edema was found in 18 cases of RVO. Triglycerides and LDL were found to be elevated in more than $50 \%$ of the cases. Macular edema was found in 42 cases of RVO. Triglycerides, total cholesterol and LDL were found to be elevated in 50\% of these cases. Correlation of VLDL with disc edema and macular edema was found to be statistically highly significant.

- Retinal neovascularization was found in 9 cases of RVO. LDL level was found to be elevated in $55.56 \%$ of the cases and decreased HDL level was found in $44.44 \%$ of cases. Disc neovascularization was found in 2 cases, both were CRVO patients. Triglycerides, total cholesterol, VLDL were found to be elevated in one of the patients while increased LDL and decreased HDL was found in both the cases. Correlation of HDL with both retinal and disc neovascularization was found to be significant.

\section{Conclusion}

Hyperlipidemia seems to be a major risk factor for RVO.If we know the major categories of dyslipidemia and its effects on the eye, we can have a well-reasoned plan of action for dealing with each one, and know when to refer to a specialist. Most of the studies imply the importance of hyperlipidemia in the pathogenesis of RVO. It remains to be determined whether lowering of serum lipids can decrease the incidence, progression and possible complications of RVO.

\section{References}

[1]. Kirwan JF, Tsaloumas MD, Vinall H, Prior P, Kritzinger EE, Dodson PM. Sex hormone preparations and retinal vein occlusion. Eye (Lond) 1997; 11: 53-6.

[2]. Hayreh SS, The ophthalmic a, 3 Branches Br. J Ophthalmology, Vol. 1, page no. 56-59.

[3]. Myron Yanoff, Jay S Duker (2009); Ophthalmology ( $4^{\text {th }}$ edition) Elsevier, page 526-531. Oh DE, Park SE, Lee JH., J Korean Ohthalmol Soc.2003 Aug 44 (8): 1806-1812.

[4]. Rymarz E, Matysik-Woźniak A, Baltaziak L, Prystupa A, Sak J, Grzybowski A. Lipaemiaretinalis an unusual cause of visual acuity deterioration. MedSciMonit. 2012 Aug; 18 (8): CS72-75. 